\title{
Framework for a Bayesian Network Version of IDHEAS
}

\author{
K. Zwirglmaier \& D. Straub \\ Engineering Risk Analysis Group, Technische Universität München, Germany \\ K.M. Groth \\ Sandia National Laboratories, Albuquerque, New Mexico
}

\begin{abstract}
Bayesian networks (BNs) have been identified as a powerful tool for human reliability analysis (HRA), with multiple advantages over traditional HRA methods. We present a framework for developing a BN version of the IDHEAS (Integrated Decision-Tree Human Event Analysis System) HRA method, which is currently under development at the US NRC. The framework includes an extension of the IDHEAS graphical model to include additional causal paths and the use of $\mathrm{BN}$ node reduction algorithms to facilitate quantification of the model. The proposed framework provides the foundation to resolve several needs frequently expressed by the HRA community. Firstly, the developed extended BN structure illustrates the causal paths found in cognitive psychology literature, thereby addressing the need for causal traceability and strong scientific basis. Secondly, by using node reduction algorithms, the BN can be reduced to a level of detail at which quantification is as straightforward as in the original IDHEAS approach.
\end{abstract}

\section{INTRODUCTION}

For many complex engineering systems a comprehensive probabilistic risk assessment (PRA) is an essential element of safety and reliability assurance. A PRA should answer the questions: What are possible failure scenarios? What are corresponding adverse consequences? What are the failure scenarios' probabilities? Many engineering systems can be characterized as human-machine systems, meaning that the human operator and the technical system are interacting. For that reason it is essential for a PRA to consider not only failures of technical components but also the effect of human actions as well as human inaction. Human reliability assessment (HRA) models human elements as part of PRAs through identification and quantification of human failure events (HFEs) in PRA models. A variety of methods have been developed and applied in this field to determine human error probabilities (HEPs) corresponding to HFEs. Among the most important representatives are THERP (Swain and Guttmann, 1983), SPAR-H (Gertman et al., 2005) and ATHEANA (Cooper et al., 1996).

The limitations of existing HRA methods are widely known (Woods, 1990, Hollnagel, 2000, Mosleh and Chang, 2004, Sträter, 2004, Boring et al., 2007, French et al., 2011, Groth and Swiler, 2013). Two interrelated shortcomings of in existing HRA methods are the limited technical and scientific basis used to develop those methods and the use of overly sim- plified modeling techniques (lacking causal structure and quantitative traceability).

To address some of the limitations in the technical basis of older HRA models, the U.S. Nuclear Regulatory Commission (NRC) has initiated development of a new HRA method called IDHEAS (Integrated Decision-Tree Human Event Analysis System)(Xing et al., 2013). The new method strengthens part of the technical basis of HRA through its foundation in psychological literature (Whaley et al., 2012). However, the new method uses overly simple quantitative models, and as a result falls short in capturing causal relationships and the complexity of human-machine interactions.

These shortcomings have been echoed in comments from the NRC ACRS, which has identified two major shortcomings of the quantification method (Stetkar, 2014). Firstly, it remains unclear why specific cognitive mechanisms and PIFs (performance influencing factors), which were identified as part of the cognitive foundation in (Whaley et al., 2012), were not explicitly considered in the IDHEAS crew failure modes (CFMs). Secondly, a "formal and complete expert elicitation process [...] to develop human error probabilities and associated uncertainty distributions for each combination of contextual factors" (Stetkar, 2014) is lacking. In this paper, we propose a methodology to address both of these shortcomings. We propose a methodology to develop a Bayesian network (BN) model for a CFM step by step, starting from the corresponding IDHEAS decision tree model, expanding it to a level where its 
cognitive foundation is modeled explicitly, and finally reducing the expanded model to a level where its quantification becomes straightforward. This process enhances the traceability between the IDHEAS quantification models and the underlying cognitive literature basis.

This paper also briefly touches upon the quantification of the model by expert elicitations in combination with a database. The final model is therefore based on both expert knowledge and observed data. While experience has shown that it is almost impossible to get full agreement in an expert elicitation process, data-scarcity in HRA applications prohibits a quantification fully based on past data. However, since both experts and data provide a valuable source of information, a reliable HRA model should be based on a combination of them.

\section{BAYESIAN NETWORKS}

Like decision, event and fault trees, which are well known in the HRA community, BNs are a probabilistic modeling tool that is compatible with PRAs. The graphical or qualitative part of a $\mathrm{BN}$ can be seen as a documentation of the causal dependencies between the random variables included in the model. A key advantage of BN models is that they can explicitly represent the causality among the variables in the model. The efficiency of BNs for quantification is based on independence assumptions that ideally follow from a causal model. Only a short introduction to the most important technical aspects of BNs is provided here. For a more in-depth treatment of BNs, the reader is referred to textbooks (Jensen and Nielsen, 2007, Kjaerulff and Madsen, 2013).

BNs are an efficient representation of a joint probability distribution $p(\boldsymbol{Z})$ over a random vector $\boldsymbol{Z}$, by graphical means. Each node represents a random variable $Z_{i}$. The qualitative dependence structure between the random variables $Z_{i}$ is represented by a directed acyclic graph (DAG). Family terms are used to describe relationships between random variables in a $\mathrm{BN}$ e.g., in Fig $1, Z_{5}$ is a child of $Z_{2}, Z_{3}$ and $Z_{4}$, which in turn are its parents, $p a\left(Z_{5}\right)$. Furthermore $Z_{1}$ to $Z_{4}$ are ancestors of $Z_{5}$, and $Z_{5}$ is a descendant of the former ones. Interpreting the $\mathrm{BN}$ in Fig. 1 casually it can be said that $Z_{1}$ directly influences $Z_{3}$ and $Z_{4}$, but only indirectly influences $Z_{5}$ through $Z_{3}$ and $Z_{4}$.

Marginal probability distributions are attached to nodes without parents, whereas conditional distributions are attached to nodes with parents. We restrict ourselves to BNs with discrete random variables. In this case the distributions are represented in conditional probability tables (CPTs).

With the independence assumptions encoded in the $\mathrm{BN}$ graph, the well-known chain rule in Eq. 1 reduces to the expression in Eq. 2 for a BN.

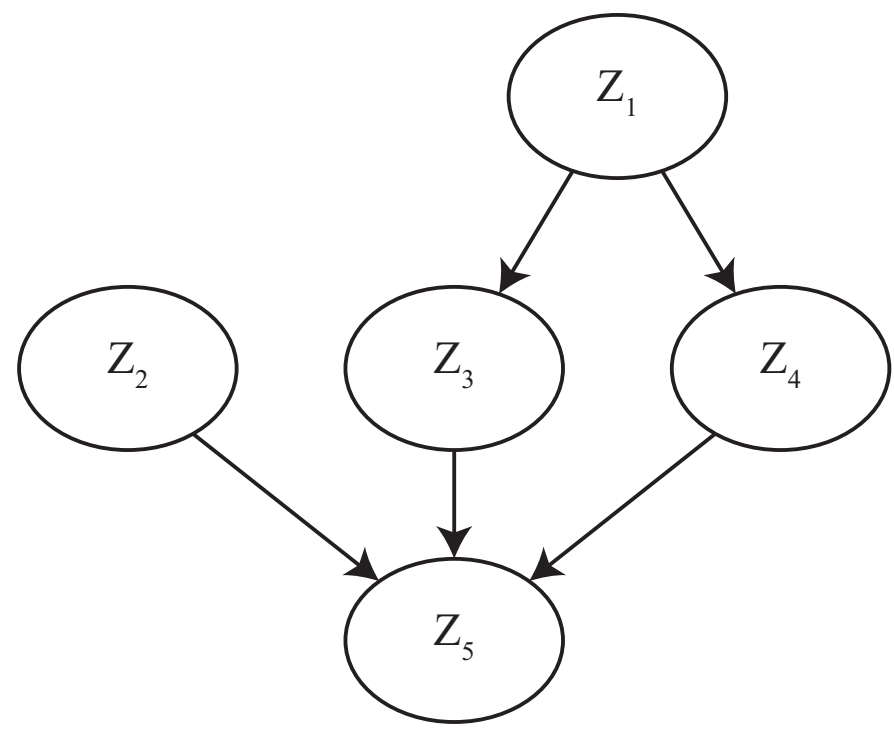

Figure 1. Example BN structure documenting the causal relationships between five variables (Z1 through $Z 5$ ). In an HRA application, this model could be interpreted as the relationship between four PSFs $(Z 1, Z 2, Z 3, Z 4)$ and error (Z5)

$\begin{aligned} p(\mathbf{z})=p\left(z_{n} \mid z_{n-1}, \ldots, z_{1}\right) & \\ \cdot & p\left(z_{n-1} \mid z_{n-2}, \ldots, z_{1}\right) \cdot p\left(z_{1}\right)\end{aligned}$

$p(\mathbf{z})=\prod_{i=1}^{n} p\left(z_{i} \mid p a\left(Z_{i}\right)\right)$

The advantage of this reduction is that it facilitates the quantification of the model by separating conditionally independent terms; it is significantly easier to elicit or quantify reduced terms, e.g., $p\left(z_{4} \mid z_{1}\right)$ vs. $p\left(z_{4} \mid z_{3}, z_{2}, z_{1}\right)$. The gain in efficiency through the use of BNs depends on the links and therefore on the independence assumptions made. Furthermore the number of parameters needed to define the CPT of a node $Z_{i}$ depends on the number of parents of $Z_{i}$ and on the number of states of $Z_{i}$ and its parents. The BN model allows practitioners to conduct reasoning about variables in the model (e.g., the HEP given the states of some or all PIFs).

\section{IDHEAS}

The IDHEAS method is being developed to address an NRC need for a new HRA model, which is "practical and straightforward to use" and which "integrates the good features in HRA state-of-practice methods and incorporate[s] the state of knowledge on human performance and cognitive psychology“ (Xing et al., 2013). 


\subsection{IDHEAS modeling approach}

For each HFE modeled in the PRA, the HRA procedure with IDHEAS entails several activities:

- Performing a qualitative task analysis and documenting crew failure paths in a crew response tree (CRT).

- Selecting applicable crew failure modes (CFMs) for each event CRT.

- Quantifying the individual CFMs via DTs and combining probabilities of the relevant CFMs to calculate the human error probabilities (HEPs) for each event.

- Analyzing HFE dependency analysis and possible recovery actions.

Here we focus specifically on the modeling and quantification of the CFMs. The method employs 14 CFMs representing failures that are typical for human performance in nuclear power plant control rooms. Each CFM is quantified using a decision tree $^{1}$ (DTs) e.g., Fig 2. The probability of occurrence of a CFM depends on the circumstances under which the tasks have to be performed. IDHEAS, like most other HRA methods, uses performance influencing factors (PIFs) - also called performance shaping factors (PSFs) - to characterize the circumstances or context. For each CFM, relevant PIFs were identified, based on a review of the cognitive basis report. Each PIF is represented as a branch point in the DT. For simplicity, the IDHEAS developers chose to limit the number of PIFs in each DT to four.

\subsection{Critical data misperceived crew failure mode}

In the remainder of this paper the CFM 'critical data misperceived' is used exemplarily to demonstrate the proposed framework. For that reason this CFM is presented to some detail in the following. 'critical data misperceived' captures situations where, e.g., a parameter has to be read from a control panel or the status of some piece of equipment is to be determined from an indication on the control panel and this piece of information is critical in the sense that it will lead to an incorrect response (Xing et al., 2013). Three PIFs are used to describe the context: HSI (human-system interface)/environment, workload, and training ${ }^{2}$. All the PIFs are binary with

\footnotetext{
${ }^{1}$ Note: The used models are referred to as decision trees in the IDHEAS report (Xing et al, 2013). However since there are no decisions involved, the tool should rather be termed event tree in a PRA sense. Since this paper is mainly intended for the HRA community we stick to the terms used in the IDHEAS report.

${ }^{2}$ This DT also contains a branch for recovery potential, which is used in most IDHEAS CFM. However, "recovery potential" has not been clearly defined in IDHEAS, and thus we neglect this concept in the remaining sections of the paper.
}

states labeled as (poor and good), (high and low) or (no and yes) respectively. In Fig. 2 the DT for the CFM 'critical data misperceived' is shown. Each path through the DT represents one possible crew failure scenario. The analysts are provided with a set of two to five questions for each PIF guiding them in determining the states of the PIFs. Expert elicitation was used to assign probabilities to the event of misperception of critical data given each combination of PIFs.

\section{DEVELOPMENT OF A CAUSAL BN STRUCTURE FOR THE CFM}

For each CFM the DT can be transformed to a BN. Assuming that the PIFs are independent, if there is no information on the state of the target node, the BN structure consists of the CFM node - in our case 'Misperception of critical data' - which has all the PIFs as its parents. Apart from that the PIFs are unconnected. Such a BN reveals little about the causal background of a HRA method. For that reason we develop an expanded BN structure for the CFM,

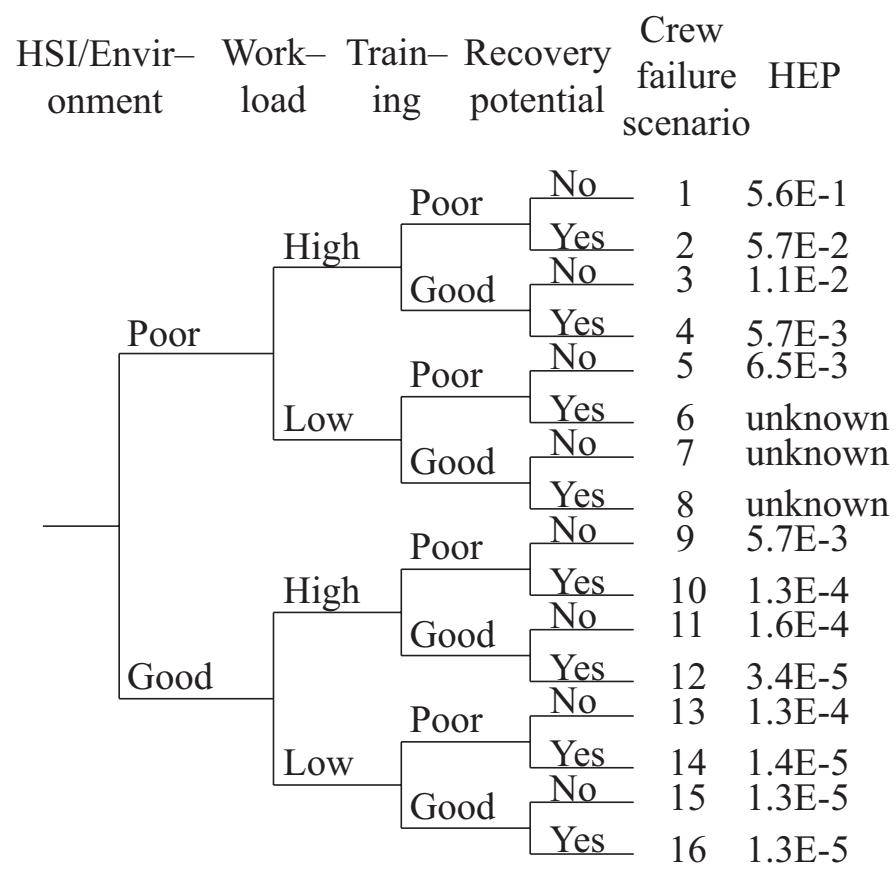

Figure 2. Decision Tree (DT) for the CFM 'Critical data misperceived'. E.g., the HEP for Poor HSI/Environment, High Workload, Poor Training and No Recovery potential is 0.56 .

which documents the cognitive psychological foundation of the method.

\subsection{Expanded BN structure}

The model contains an additional layer of nodes (white in Fig. 3), which are intended to specify the PIFs that are often too generic or abstract for ana- 
lysts to directly determine their states, but which are critical for understanding of human performance.

IDHEAS provides questions and rules for the analysts to consider when determining the states of the PIFs. The additional layer of PIF specification nodes can thus be based on these questions (light grey in Fig 4). The literature serving as a foundation for IDHEAS (Whaley et al., 2012) summarizes the causal paths that could lead to a crew failure event based a comprehensive study of cognitive psychology. These paths may be implemented directly in the model to add additional causal details extracted from scientific literature. For the example CFM (Fig. 3) there a three main causal paths stemming from cognitive literature:
- The first causal path (path I in Figure 3) is the misperception of data due to extreme HSI/Environment conditions. E.g., the quality of the HSI or factors in the environment degrade the information in such a way that it is misperceived; technically this could be seen as an instrumentation failure rather than a human error, but this instrumentation failure would manifest as a human failure event.

- The second causal path (path II in Figure 3) leading to misperception of data is from attention degradation. Attention can be degraded due to a combination of factors, including characteristics of the situation and the information (e.g., the HSI and environment), high workloads, multiple priorities

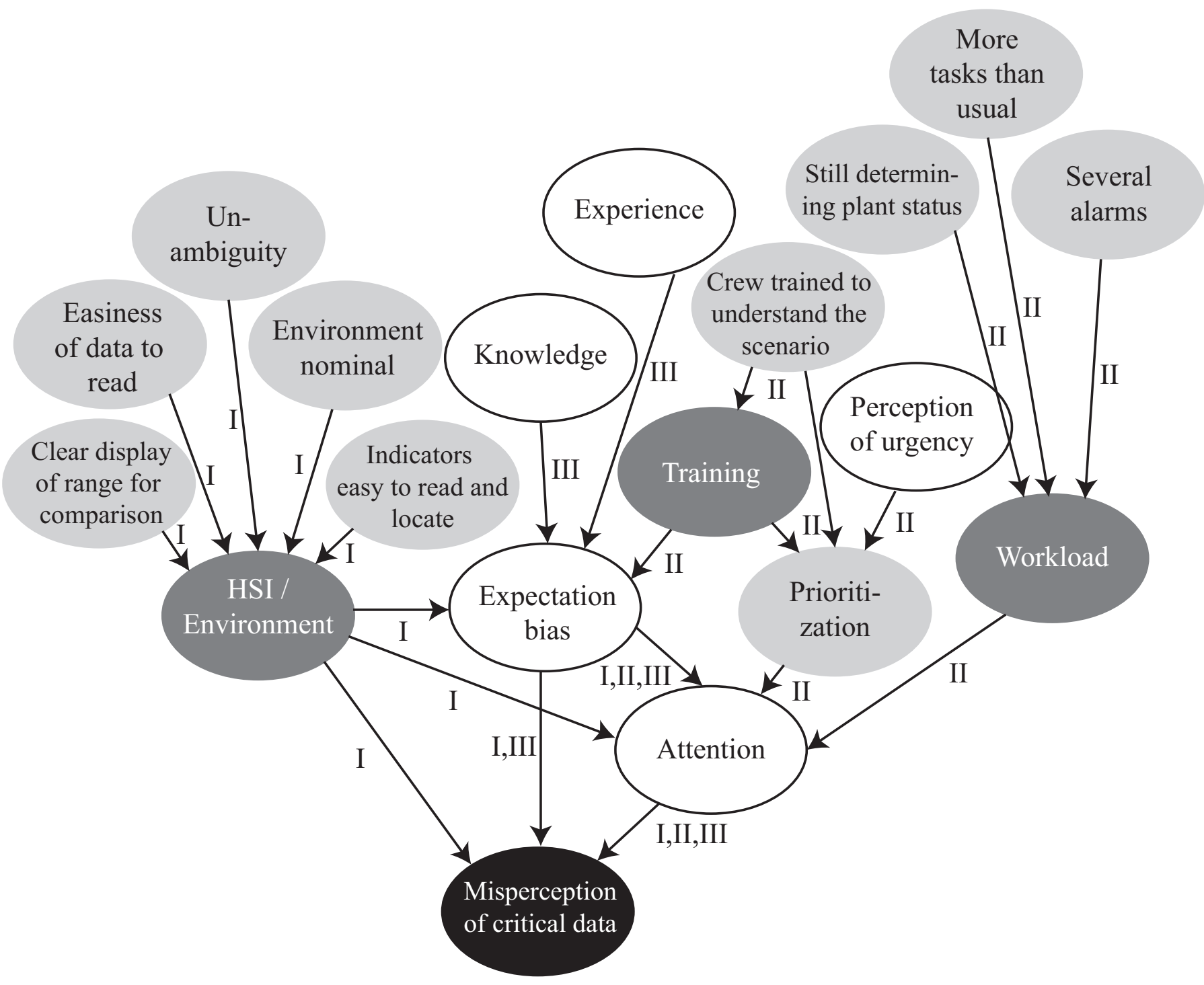

Fig 3. Fully expanded BN structure revealing the cognitive psychological background of IDHEAS. The numbers (I-III) show the failure paths, which may lead to Misperception of critical data. 
and through biases introduced by training, knowledge, and experience. Training, workload and perception of urgency cause the crew to prioritize certain tasks, alarms etc. and direct attention resources to high priority tasks. A misdirection of attention could lead to misperception of the critical data. The prioritization and the crew members' expectation biases determines the amount of attention paid to the various pieces of information, which again may lead to misperceiving the critical data (paths I in Fig. 3).

- The third causal path (path III in Figure 3) stems from expectation biases leading to misperception. Experience and knowledge may introduce an expectation bias, which again may directly cause misperception of critical data (e.g., situations where a person "sees what they want to see") or indirectly through changing the person's attention (path II).

As shown in the model, the PSFs identified in the IDHEAS model influence the occurrence of CFM through multiple causal paths. HSI/Environment influences CFM through one direct causal path and additionally through two indirect causal paths. Training also influences CFM (indirectly) through two different causal paths. One causal path, expectation bias, is only indirectly captured in the original IDHEAS model.

The IDHEAS questions (light grey nodes) are intended to determine relevant aspects associated with the three PIFs, and are used here to demonstrate how observable questions can be explicitly included in the model. In some cases (prioritization) the nodes based on the IDHEAS questions also provide a critical causal detail.

\subsection{BN structure for IDHEAS-like quantification}

The full model in Fig 3 can be quantified using a variety of approaches. However, a main objective of this work is to develop a HRA model based on IDHEAS, and thus to limit the amount of additional information that must be elicited. To achieve this goal, the model in Fig 3 is reduced to a form that closely resembles the original IDHEAS DT augmented with the DT questions from (Xing et al., 2013). To do so, the node removal algorithm (Shachter, 1986, Shachter, 1988, Straub and Der Kiureghian, 2010) are applied. This algorithm allows removing nodes, which have not received evidence, in a way that the independence assumptions incorporated in a BN are not altered. The two principals of node removal are: First a node, which has not received evidence and which does not have children can be removed from the network; Secondly the direction of a link between two nodes $Z_{i}$ and $Z_{j}$ can be reversed if $Z_{i}$ inherits $Z_{j}$ 's parents and vice versa and if this does not cause the $\mathrm{BN}$ structure to become cyclic.

Reducing the white nodes from the structure in Fig. 3 leads to the structure in Fig. 4. In this structure Prioritization is directly connected to the target node. The causal interpretation of this would be that workload may lead to a larger HEP, but only if the crew does not set priorities correctly. Since this would introduce a new PIF to the CFM, and we aim at keeping the quantification of the model as close to the original method as possible, we indicate this only qualitatively in the model.

The BN structure in Fig. 4 has significant advantages over the IDHEAS DT or its equivalent BN structure. Firstly, in this model the analyst would directly answer the PIF questions rather than assigning a PIF state based on implicit consideration of the questions, which is much more abstract ${ }^{3}$. The explicit inclusion of PIF questions in the model, which expands the level of documentation provided by the model, and enhances the traceability from analysis input to probability estimate, and reduces variability between analyst

Secondly if marginal probabilities are elicited for the PIF specification nodes, as was done in (Hallbert and Kolaczkowski, 2007), the BN in Fig. 4 can deal with missing information or uncertainty about one of the PIF specification nodes' states. For example, the HRA analyst may lack information about specific indicator designs, which may make it difficult to assess the 'easiness of data to read. In situations where the analyst does not have information about one or more PIFs, the analyst can use the prior probabilities in the $\mathrm{BN}$ rather than guessing or making unwarranted assumptions about the system.

Thirdly, the fully quantified $\mathrm{BN}$ can be used to reason about additional problems and gather additional insight. With identical analyst inputs, the BN structure in Figure 4 will produce the same HEP assignments as the IDHEAS DT. The BN structure also offers the opportunity to reason about the PIFs, given knowledge of the CFM (and/or other PSFs). This provides a powerful benefit: the ability to identify which PSFs (or PSF details) states are likely to be present when we know there is an error. This provides powerful insight into the probabilities of the causes or errors, which is a critical piece of information that can be used to prevent errors. Additional insight into this topic are provided in (Groth and Swiler, 2013).

\footnotetext{
${ }^{3}$ This is also true if the original DT is considered together with the questions provided in the IDHEAS report
} 


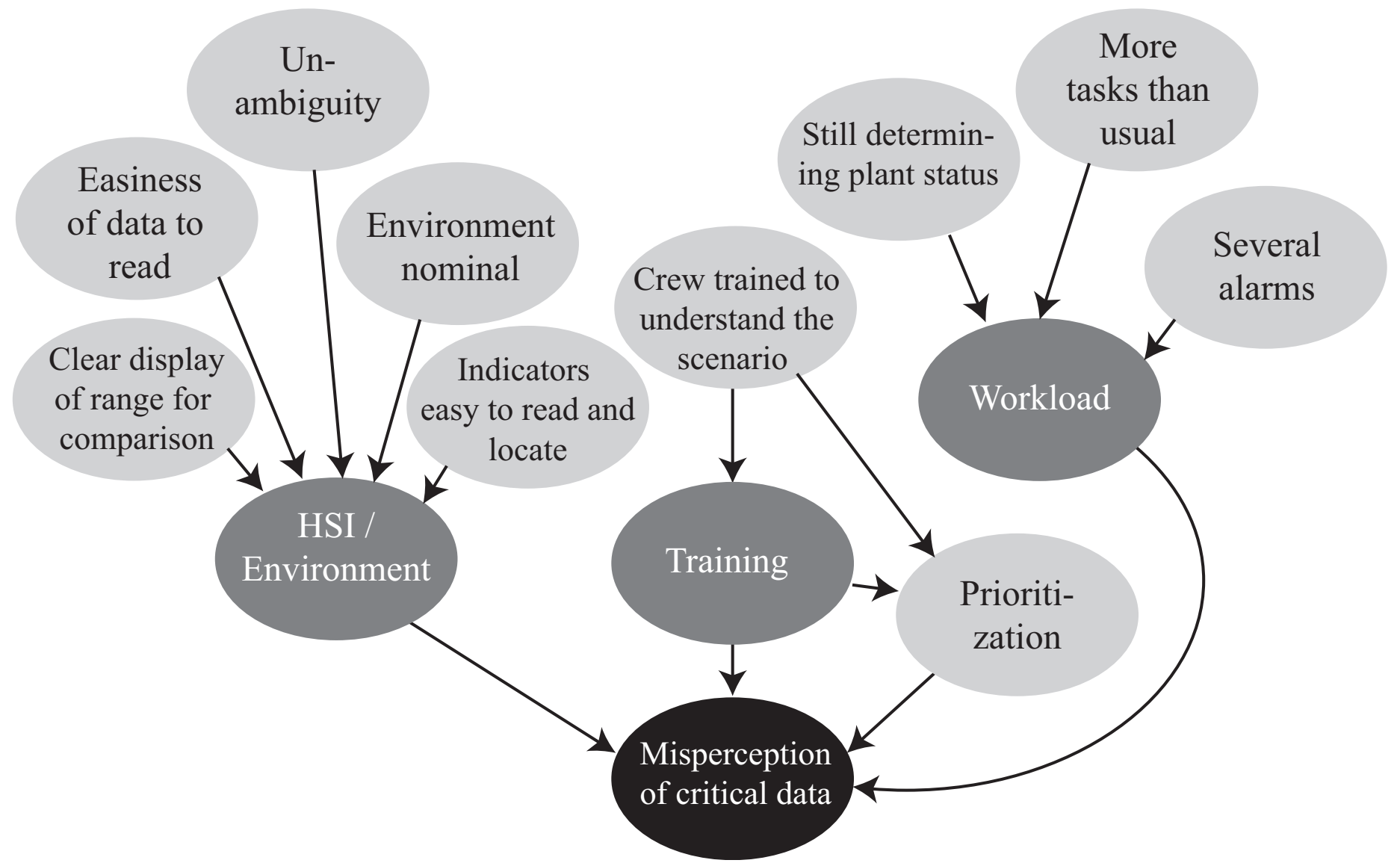

Figure 4. Reduced BN structure for IDHEAS-like quantification.

\section{A CONCEPT FOR MODEL QUANTIFICATION}

Once the qualitative dependence structure between the random variables in the model has been developed, these dependencies are to be quantified. In this section we briefly sketch the approach for quantifying the BN structure in Fig. 4. For brevity we skip the numerical results in this paper and refer to (Zwirglmaier et al., 2015). The PIF specification nodes are quantified through expert elicitations. The states of the PIFs conditional on the states of the PIF specification nodes can be determined through deterministic rules given in the IDHEAS report (Xing et al., 2013). Furthermore, the same report provides HEPs for the crew failure scenarios. These numbers are elicited from experts. In the scope of these elicitations experts additionally provide uncertainty estimates in the form of quantiles. We use these estimates for the quantification of the IDHEAS-BN. However, since sound models should be based on all information available, we apply Bayesian updating to enhance the model. Bayesian updating for HRA is described in (Groth et al., 2014).
From the quantiles elicited in (Xing et al., 2013), we take the 0.1 and 0.9 quantile of the HEP of a crew failure scenario $i$ (here denoted as $\theta_{\mathrm{i}}$ ). A beta priordistribution with PDF $\pi_{0}(\theta)$ is fitted to these quantiles:

$$
\pi_{0}\left(\theta_{\mathrm{i}}\right)=\frac{1}{B\left(a_{0}, b_{0}\right)} \theta^{a_{0}-1}(1-\theta)^{b_{0}-1}
$$

denotes the beta function and $a_{0}$ and $b_{0}$ are the parameters of the distribution. Assume now a database, where $n$ cases of crew failure scenario $i$ are reported, of which $n_{e}$ resulted in a HFE. This information can be used to update our belief about the HEP associated to this crew failure scenario. The updated belief is described through a beta posterior distribution $\pi_{1}\left(\theta_{\mathrm{i}}\right)$ with parameters $a_{1}$ and $b_{1}$ i.e.:

$$
\begin{aligned}
& a_{1}=a_{0}+n_{e} \\
& b_{1}=b_{0}+\left(n-n_{e}\right)
\end{aligned}
$$




\section{DISCUSSION}

This paper presents a framework for the application of BNs to address many shortcomings of the IDHEAS HRA-method. Multiple research groups have shown that BNs can provide a sound and versatile mathematical model to mitigate many of the shortcomings of older HRA methods (Baraldi et al., 2009, Mosleh et al., 2012, Li et al., 2012, Groth and Swiler, 2013, Ekanem and Mosleh, 2014, Mkrtchyan et al., 2015).

Causal traceability is a major need of HRA methods. In this paper we propose to have an expanded $\mathrm{BN}$ structure qualitatively revealing the theoretical background of the method and a reduced structure, which enables a more straightforward quantification than the full expanded structure. While both structures are quantifiable from a mathematical point of view, quantification of the expanded structure is difficult from a HRA-perspective because of lack of data and the difficulty in estimating the required probabilities by experts. If the expanded BN was implemented in a software tool, the additional nodes from the expanded structure could for example be presented in a lighter color, showing that these nodes are necessary for the understanding of the causal relationships but are not quantified. While most of the developed methods have a strong background from the psychological side, this background is usually hidden to more applied users, who are presented with only a reduced number of PIFs. By developing expanded BN structures and presenting them to users, the theoretical background becomes more traceable even if it may not be possible to present it in full detail in this manner. It has been found by many researchers that in HRA the results depend strongly on the person doing the analysis e.g., (Lois et al., 2009). This can be seen as a major point of criticism against the HRA methods. Granting the users of the methods more insight may not completely overcome this problem, however it will certainly help to mitigate it.

Another major need in HRA, which is only briefly addressed here, is an exhaustive and rigorous quantification framework. It is generally known that HRA models are not capable or even intended to fully capture all aspects of human behavior. Nevertheless, it is necessary to model human error, using all information and knowledge available. While many HRA researchers rely on quantifying their models either through experts or through data, our proposed quantification framework combines these two. Combining expert knowledge and data is well in line with the Bayesian understanding of probability used throughout PRA (Kelly and Smith, 2009) and is the only method to come up with sound probability estimates in an industry with scarce data. Using Bayesian updating allows using continuously more data to update the parameters of the $\mathrm{BN}$, in order to improve the quality of the model.

A last point implicitly addressed in this paper is the applicability of BN-based HRA methods for everyday HRA practice. While HRA researchers may be tempted to embrace BNs simply for their powerful modeling features, HRA practitioners call for models that are applicable in their everyday practice. Not many of the BN HRA models developed up to this point satisfy this need. (Groth and Swiler, 2013) realized this and proposed a BN version of SPAR-H. SPAR-H is the HRA method mostly used by the United States Nuclear Regulatory Commission (NRC). By converting the widely used SPAR-H HRA method into a BN, they showed that BN-based HRA methods can readily meet the HRA practitioners' needs; it is not actually due to the BN that most BN-based HRA methods do not meet the practitioner's needs. Following the same line of thought, in this paper we propose a BN version of IDHEAS, which is capable of meeting the needs of both HRA researchers and HRA practitioners.

Since IDHEAS is still in development, it is not currently used in practice, however it is intended as a replacement for SPAR-H at the US NRC, and thus its development focuses clearly on practical usability as well as technical foundations. The IDHEAS-BN approach expands these technical foundations with additional rigor beyond the DTs in the original version of IDHEAS. As illustrated in this work, the IDHEAS-BN demonstrates can be both wellgrounded in science and practical for use by HRA practitioners. In the scope of this paper we propose a framework for modeling and quantifying the IDHEAS decision trees in a BN environment. We illustrate the process using a single CFM. Further research is necessary to apply the proposed framework to the remaining CFMs and to carry out more detailed expert elicitations etc.

\section{CONCLUSION}

This paper proposes a framework for treating the IDHEAS HRA method in a Bayesian network environment. We base the work on an existing HRA method, which is intended for practical use. By doing so the need for practically applicable methods is satisfied. Furthermore we address the needs from the HRA community for a well-founded quantification framework and causal traceability within the HRA methods.

\section{REFERENCES}

BARALDI, P., CONTI, M., LIBRIZZI, M., ZIO, E., PODOFILLINI, L. \& DANG, V. N. 2009. A Bayesian Network Model for Dependence 
Assessment in Human Reliability Analysis. ESREL 2009. Prague.

BORING, R. L., GRIFFITH, C. D. \& JOE, J. C. The Measure of human error: Direct and indirect performance shaping factors. Human Factors and Power Plants and HPRCT 13th Annual Meeting, 2007 IEEE 8th, 26-31 Aug. 2007 2007. 170-176.

COOPER, S. E., RAMEY-SMITH, A. M., WREATHALL, J., PARRY, G. W., BLEY, D., LUCKAS, W. J., TAYLOR, J. H. \& BARRIERE, M. T. 1996. A Technique for Human Error Analysis (ATHEANA).

EKANEM, N. J. \& MOSLEH, A. 2014. Phoenix - A Model-Based Human Reliability Analysis Methodology: Quantitative Analysis Procedure and Data Base. PSAM 12. Honolulu.

FRENCH, S., BEDFORD, T., POLLARD, S. J. T. \& SOANE, E. 2011. Human reliability analysis: A critique and review for managers. Safety Science, 49, 753-763.

GERTMAN, D., BLACKMAN, H., MARBLE, J., BYERS, J. \& SMITH, C. 2005. The SPAR$\mathrm{H}$ Human Reliability Analysis Method. Idaho National Laboratory.

GROTH, K. M., SMITH, C. L. \& SWILER, L. P. 2014. A Bayesian method for using simulator data to enhance human error probabilities assigned by existing HRA methods. Reliability Engineering \& System Safety, 128, 32-40.

GROTH, K. M. \& SWILER, L. P. 2013. Bridging the gap between HRA research and HRA practice: A Bayesian network version of SPAR-H. Reliability Engineering \& System Safety, 115, 33-42.

HALLBERT, B. \& KOLACZKOWSKI, A. 2007. The Employment of Empirical Data and Bayesian Methods in Human Reliability Analysis: A Feasibility Study.

HOLLNAGEL, E. 2000. Looking for errors of omission and commission or The Hunting of the Snark revisited. Reliability Engineering \& System Safety, 68, 135-145.

JENSEN, F. V. \& NIELSEN, T. D. 2007. Bayesian networks and decision graphs, New York [u.a.], Springer.

KELLY, D. L. \& SMITH, C. L. 2009. Bayesian inference in probabilistic risk assessmentThe current state of the art. Reliability Engineering \& System Safety, 94, 628-643.

KJAERULFF, U. B. \& MADSEN, A. L. 2013. Bayesian Networks and Influence Diagrams: A Guide to Construction and Analysis, Springer Publishing Company, Incorporated.

LI, P.-C., CHEN, G.-H., DAI, L.-C. \& ZHANG, L. 2012. A fuzzy Bayesian network approach to improve the quantification of organizational influences in HRA frameworks. Safety science, 50, 1569-1583.

LOIS, E., DANG, V. N., FORESTER, J., BROBERG, H., MASSAIU, S., HILDEBRANDT, M., BRAARUD, P.,
PARRY, G., JULIUS, J., BORING, R. L., MÄNNISTÖ, I. \& BYE, A. 2009. International HRA Empirical Study - Phase 1 Report: Description of Overall Approach and Pilot Phase Results from

Comparing HRA Methods to Simulator Performance Data. Washington D.C.: US Nuclear Regulatory Commission.

MKRTCHYAN, L., PODOFILLINI, L. \& DANG, V. N. 2015. Bayesian belief Networks for Human reliability analysis: a review of applications and gaps. Reliability Engineering \& System Safety.

MOSLEH, A. \& CHANG, Y. H. 2004. Model-based human reliability analysis: prospects and requirements. Reliability Engineering \& System Safety, 83, 241-253.

MOSLEH, A., SHEN, S. H., KELLY, D. L., OXSTRAND, J. H. \& GROTH, K. 2012. A Model-Based Human Reliability Analysis Methodology. PSAM 11. Helsinki.

SHACHTER, R. D. 1986. Evaluating influence diagrams. Oper. Res., 34, 871-882.

SHACHTER, R. D. 1988. Probabilistic inference and influence diagrams. Oper. Res., 36, 589604.

STETKAR, J. W. 2014. Human Reliability Analysis Models. Slides 15-28 from "ACRS Meeting with THE U.S. NUCLEAR REGULATORY COMMISSION October 2, 2014.”. United States Nuclear Regulatory Commission.

STRÄTER, O. 2004. Considerations on the elements of quantifying human reliability. Reliability Engineering \& System Safety, 83, 255-264.

STRAUB, D. \& DER KIUREGHIAN, A. 2010. Bayesian Network Enhanced with Structural Reliability Methods: Methodology. Journal of Engineering Mechanics, 136, 1248-1258.

SWAIN, A. D. \& GUTTMANN, H. E. 1983. Handbook of Human Reliability Analysis with Emphasis on Nuclear Power Plant Applications. Washington DC: U.S. Nuclear Regulatory Commission.

WHALEY, A., XING, J., BORING, R. L., HENDRICKSON, S., JOE, J. \& LE BLANC, K. 2012. Building a psychological foundation for human reliability analysis (NUREG-2114). Washington DC: US Nuclear Regulatory Commission.

WOODS, D. D. 1990 . On taking human performance seriously in risk analysis: Comments on dougherty. Reliability Engineering \& System Safety, 29, 375-381.

XING, J., PRESLEY, M., PARRY, G., FORESTER, J., HENDRICKSON, S. \& DANG, V. 2013. NRC/EPRI Draft Report for Peer Review: An Integrated Decision-Tree Human Event Analysis System (IDHEAS) Method for NPP internal at-power operation. NRC/EPRI

ZWIRGLMAIER, K., STRAUB, D. \& GROTH, K. 2015. Enhancing human reliability analysis by causal modeling of cognitive factors: A Bayesian network version of IDHEAS, Journal paper manuscript. 